\title{
ANALYSIS ON MECHANICAL STRUCTURE AND AUTOMATIC CONTROL OF AN INTELLIGENT LOGISTICS VEHICLE
}

\author{
Shenyi Juan ${ }^{1}$ \\ ${ }^{1}$ Business School, Wuchang University of Technology, Wuhan, 430223, China. \\ Email: juanshenyisywh@163.com
}

\begin{abstract}
In order to solve the application of driving motor drive structure and sensor technology in logistics vehicles to make the logistics vehicles adapt to more complex road conditions, a research on logistics vehicle based on electronic differential speed is presented, and its mechanical structure and automatic control system are designed. Firstly, the hub motor, mechanical structure, automatic controller, electronic differential principle, and fuzzy PI control theory of intelligent logistics vehicle are introduced. Besides, the hub motor, mechanical structure and automatic controller are designed. For the design of hub motor, the drive motor is chosen as brushless direct current (DC) motor. In the mechanical structure, three kinds of wheel structure are introduced, and the four-wheel structure is selected. The Intelligent Power Module (IPM) of IKCM30F60GA driving circuit is designed for the automatic controller. Then, the vehicle control strategy based on electronic differential velocity model system master control module algorithm is constructed. Finally, an external hub motor is constructed for finite element simulation, and the experiment is carried out for no-load, load and electronic differential steering of the external rotor. The experimental results show that the brushless DC motor is in the form of four wheels. The mechanical structure of the wheel is that the first two wheels are driving wheels and the two rear wheels are directional wheels. According to the simulation experiment, fuzzy PI has a shorter response time, stronger anti-interference and stability than conventional PI. The logistics vehicle based on electronic differential speed designed in this research can adapt to more complex road conditions, and the degree of intelligence and automation has been improved. The research provides a good guiding ideology for the development of logistics vehicles in the e-commerce industry.
\end{abstract}

Keywords: Self-Guiding Vehicle; Mechanical Structure; Automatic Controller; Hub Motor; Electron Differential Model.

\section{Introduction}

With the progress of science and technology, the Internet has developed at a very fast speed in the past decade. The e-commerce industry is a product of the Internet development process. With the rise and rapid development of the e-commerce industry, the requirements for the logistics industry are constantly rising [1]. As the traditional logistics and transportation can no longer meet the needs of the current e-commerce, people have begun to look for a new mode of transportation. Flexible transportation and automated transportation have gradually replaced the traditional logistics and transportation. The definition of automated transportation and flexible transportation is to use intelligent robots, automated processes, unmanned trucks and intelligent commodity sorting equipment to transport in the logistics vehicles to realize the automatic logistics system of logistics vehicle, it is necessary to integrate sensor technology, drive motor technology, signal processing technology and other technologies [2].

At present, Automated Guided Vehicles (AGV) are the most representative of logistics Vehicles. The car can carry out the logistics goods according to the pre-set route, which is intelligent and doesn't need any manual operation. With the rapid development of technologies related to intelligent robots (artificial intelligence technology, sensor technology and measurement and control technology), higher and newer requirements have been put forward for AGV's autonomous judgment ability, autonomous recognition ability and obstacle recognition performance [3]. Since the existing artificial intelligence technology can't meet the demand of intelligent control logistics vehicle, this technology is also a key technology for AGV development, so it is necessary to improve the artificial intelligence control method. At present, there are three modes of automatic guide vehicle, namely manual mode, semiautomatic mode and automatic mode. 
In manual mode, the position of the automatic guide vehicle can only be observed to change in the monitoring interface; traffic management rules are included in semi-automatic mode, so the auto-guide vehicle can perform tasks in the traffic management system; the fully automatic mode refers to the management, monitoring system scheduling and control and task delivery of the automatic guide vehicle. Among the three modes, the fully automatic mode is a trend in the development of automatic guide vehicle [4]. Relevant studies have also shown that improving the autonomous judgment ability of the automatic guide vehicle and relying on its own mode to identify obstacles can contribute to the global control information [5]. Therefore, based on the analysis of the autonomous execution of the automatic guidance vehicle, it can be concluded that sensor sensing of the external environment can provide a good guarantee for the safe arrival of AGV. It is of great significance for $A G V$ to realize intelligence to analyze the external environment first and then understand the environment in an appropriate form. And compared with the wired drive, wireless technology can carry a generator over the sound of a car, increasing battery capacity. Therefore, improving the efficiency and reliability of AGV energy supply is the basis for the wide application of intelligent logistics vehicles.

To sum up, in order to improve the performance of all aspects of the adaptive guide car, the hub motor, mechanical structure and automatic controller of the automatic guide car are designed in terms of hardware. In terms of software, an algorithm based on electronic differential model system module is proposed to improve the controller. Finally, the results of no-load, load and electronic differential steering of the external rotor brushless DC motor are analyzed by simulation test. It is expected that the research can help to further optimize and improve the performance of automatic guide vehicle.

\section{Literature Review}

With the development of the logistics industry, the ecommerce industry has higher and higher requirements for logistics and transportation. Efficient transportation efficiency is the demand of the current era. In order to adapt to the high efficiency of logistics and transportation, scholars began to carry out research on this subject. Initially, researchers focused their research on logistics vehicles in order to automate them. Gao et al. (2015) combined the advantages of the automatic guide vehicle and mechanical arm in traditional logistics, designed the automatic guide logistics platform with mechanical arm, and completed the development of the prototype. The main body of the platform and the detailed structure of the manipulator are designed.
The platform, with Programmable Logic Controller (PLC) and touch screen as the core, constructs the hardware and software of the federated control system for platform automatic boot and manipulator operation, and explains the system control logic in detail. Experimental improvements have been made to the configuration of existing automatic guide vehicles, and object maintenance functions have been provided for them, which has certain practical value for expanding the scope of work and improving the automation and intelligence degree of logistics equipment [6]. Peng et al. (2016) studied the mechanical design and control system of a material transportation mobile robot. The mechanical structure uses a four-wheel drive chassis with omni-directional mobility and a damped suspension mechanism. The Siemens s7-1217c PLC controller with extended modules is used for lower level control of the robot, while the laptop is used for upper level control. The study also develops control algorithms for robot motion patterns and tests the motion control performance based on its internal triaxial potentiometer. Finally, a visual navigation algorithm based on Hough transform is designed, and experiments on the robot show that material transfer and motion control of the robot can be realized [7]. With the development of control science and sensors, different sensors are applied to the automatic guide vehicle to realize more functions of the logistics vehicle. According to the requirements of logistics vehicles, Zhang et al. (2018) designed an intelligent mechanical structure for automatic tracking guide vehicle, which is composed of clamping device, control, device drive gear and chassis. The hardware control system consists of Arduino microcontroller, tracking sensor, color sensor and infrared optoelectronic sensor. The software system includes tracking module, grasping module, color recognition module and classification module, which are controlled by Arduino MCU. Experimental results prove that, based on personalized design and target requirements, the software and hardware system designed in this research can automatically realize the tracking, carrying, classification and storage functions of commodities [8]. Wu et al. (2018) carried out structural optimization and load analysis for the heavy-duty automatic guide vehicle, aiming to improve the dynamic response performance and energy utilization efficiency of the AGV frame. Mechanical and geometric constraints are first defined to optimize the structure of the AGV framework. Secondly, the multi-objective optimization function is established for the structure size, and then the optimal solution is solved by genetic algorithm. Thirdly, according to the optimized structure size parameters, the design scheme of AGV frame is improved. Finally, ANSYS software is used to verify the stiffness and strength of the frame. 
The results show that the mass and moment of inertia of the frame are reduced by $33.7 \%$ and $19.3 \%$ respectively, and the friction of AGV wheels is reduced by $16.8 \%$. A light mechanical base is provided for motion control of a heavy AGV [9].

To sum up, there are many researches on the mechanical structure and automatic control of the automatic guide vehicle. Most of the research has been done through different methods to improve the intelligent sensing technology, the ability to recognize the environment, and the accuracy and reliability of the track.

The automatic guide vehicle is more intelligent and advanced in positioning and information processing. In addition, there are a few studies to optimize the power supply of AGVs.

In order to obtain intelligent, automated, and sensitive logistics vehicles, a new type of electronic differential speed is adopted to control the steering of the automatic guide vehicle, and the wheel speed is controlled through an external motor to make the AGV more economical and efficient.

\section{Methodology \\ 3.1 Design of hub motor for intelligent logistics vehicle}

The existing motor types include brushless DC motor, brushless DC servo motor, stepper motor and permanent magnet synchronous motor, and so on [10].

According to the advantages and disadvantages of different motor types, since the brushless DC motor as the driving motor has been able to meet the requirements of $\mathrm{AGV}$ vehicle system for control performance and driving performance, the driving motor selected in this research is brushless DC motor.

Compared with the traditional drive motor, the AGV drive system of hub motor needs to integrate transmission device, transmission and differential device, and so on.

Therefore, the external permanent magnet brushless DC hub motor is chosen as the driving motor of the AGV vehicle system designed.

The scheme has the following advantages:

I. Vehicle control mode from hard to soft connection. The speed of the wheels is consistent with that of the driving motor.

When the rotation speed is different, the vehicle controller can be used to drive each driving motor.

Different from the traditional AGV drive system, it can omit the mechanical differential gear device, transmission, transmission shaft and other parts, so that the connection between the driving car system and the wheel is more direct, which not only increases the utilization space of the vehicle, but also improves the efficiency of the vehicle.
The driving motor scheme makes the whole vehicle system drive more flexible, and increases the driving capacity (bumpy) of the logistics vehicle on the complex road surface [11].

II. The driving motor used in this research adopts the electronic differential steering technology, which can reduce the steering radius and increase the steering function of the motor.

In this research, according to the driving requirements of $\mathrm{AGV}$ vehicle system, the main parameters of the designed external motor are shown in Table 1.

Table 1. Main parameters of external motor

\begin{tabular}{|l|c|}
\hline \multicolumn{1}{|c|}{ Parameters } & Number \\
\hline Rated power/W & 250 \\
\hline Nominal voltage/V & 24 \\
\hline Rated speed/r/min & 350 \\
\hline Groove cooperating & $30 / 27$ \\
\hline Outer diameter of rotor /mm & 130 \\
\hline The axial length/mm & 35 \\
\hline
\end{tabular}

The $30 / 27$ pole-groove fit is obtained by optimizing the design. Figure 1 is the magnetic dense cloud diagram of the special motor outside the $30 / 27$ slot, and Figure 2 is the simulation diagram of the electromagnetic torque and iron consumption of the $30 / 27$ slot.

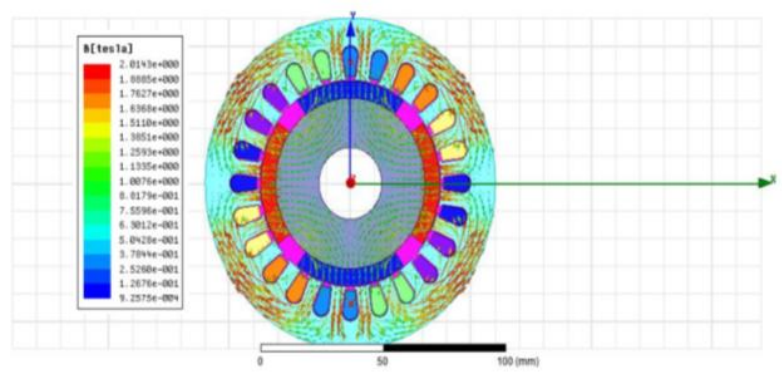

Figure 1: The simulation cloud image of magnetic field of 30/27 slot external rotating motor.

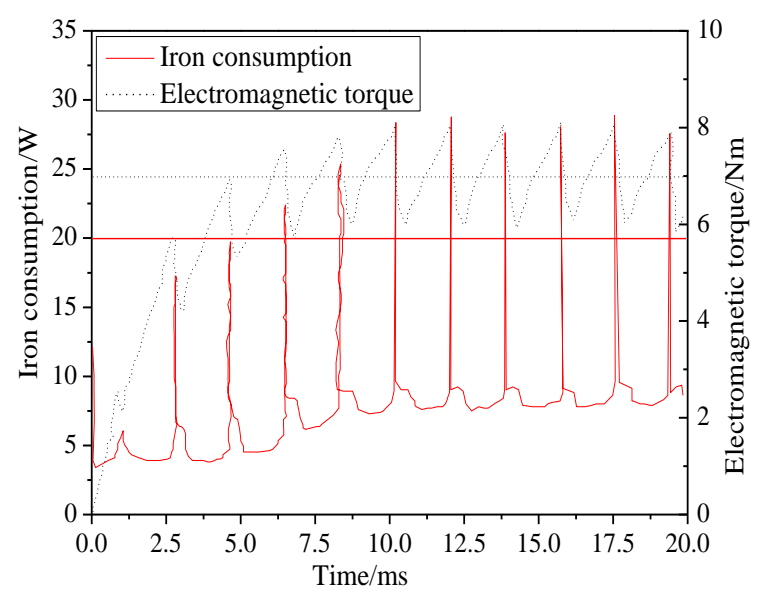

Figure 2: The simulation diagram of electromagnetic torque and iron consumption of the 30/27 slot. 
It can be concluded from Figure 1 and Figure 2 that the average electromagnetic torque at 30-pole 27 slot is $7 \mathrm{~N} \cdot \mathrm{m}$, the torque ripple is $29.3 \%$, and the average iron consumption is $20 \mathrm{~W}$, which meets the driving requirements of $\mathrm{AGV}$ designed in this research.

\subsection{Mechanical structure design of intelligent logistics vehicle}

Generally, AGV wheel system includes threewheel structure, four-wheel structure and six-wheel structure. The driving directions of AVG include oneway straight driving, two-way straight driving, insitu steering and omni-directional steering. Generally, it is needed to understand the needs of customers to design logistics vehicles.

Generally, specific wheel structure can be divided into five forms: directional wheel, drive wheel, universal wheel, steering wheel and steering drive wheel [12]. Then the design of the wheel mechanism of the logistics vehicle is introduced in detail.

First, the directional wheel. In AGV systems, directional wheels should keep the speed and motion direction of such wheels consistent with the speed and motion direction of the AGV system, and limit the lateral swing of the AGV system.

The driving wheel is an external hub motor, so the driving motor determines that the speed of the driving wheel in this research is consistent with the motion direction of the AGV system. Since the whole AGV system is powered by the driving wheel, which also determines the operating speed and kinetic energy requirements of the AGV system. For the AGV system designed in this research, at least two driving wheels are required to determine the motion direction of the AGV.

Universal wheel can conduct $360^{\circ}$ omnidirectional free steering on the ground, which is an indispensable wheel structure to realize in-situ steering. Its speed depends on the speed of the autopilot. The main function of the wheels on the autowarder is to carry weight and keep the body balanced.

The steering wheel is very similar to the universal wheel. It can also freely turn in the direction of $360^{\circ}$ without dead Angle on the ground, but the driving motor determines the steering angle of the steering Angle. Similarly, the speed of the body of the autopilot determines the speed of the steering wheel. However, the steering wheel can only guide the driving direction of the automatic guide car and can't change the driving speed of the automatic guide car. At present, the steering wheel is widely used in the front wheel of ordinary tricycle system.

Therefore, in the wheel mechanism, compared with the three-wheel form, the four-wheel form has a very large space in terms of load bearing, power function and later function addition module.
The four-wheel form for design in the whole logistics vehicle system is selected.

According to the above motor design scheme, wheel design structure and the realization of functions required by the logistics vehicle, the AGV vehicle composition frame determined in this research is shown in Figure 3.

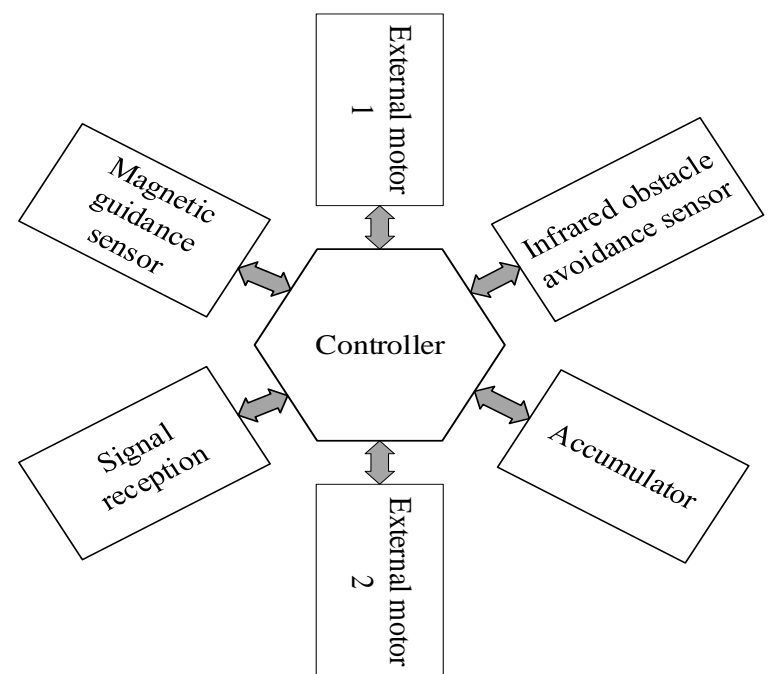

Figure 3: A frame diagram of the whole AGV vehicle.

In Figure 3 , the battery uses two $12 \mathrm{~V}$ lithium batteries in series to provide DC current for the AGV system. The battery supplies power to the whole car through the controller's power circuit. In the whole AGV, the front wheels are made up of two outwardrotating permanent magnet brushless DC hub motors, which can be used as driving wheels on one hand and steering wheels on the other.

In the whole AGV system, the controller mainly realizes two functions: one function is to receive signals to realize the drive and control of two external motors. When two external motors issue different commands, the steering can be realized. The second function is to receive and analyze the signals of the magnetic guidance sensor and the infrared obstacle avoidance sensor, so as to realize the automatic tracking and obstacle avoidance functions of AGV vehicles [13].

\subsection{Design of controller for intelligent logistics vehicle}

According to the frame diagram of the whole AGV vehicle, the controller is the core part of the whole AGV vehicle. For the design of the controller, the hardware circuit adopted is designed based on the main control chip TMS320F2808. Figure 4 is the block diagram of the controller. First, the HALL signal output in the motor is $5 \mathrm{~V}$. Before the signal is sent to the Digital Signal Processor (DSP), it should be converted to a $3.3 \mathrm{~V}$ level range with the logical information remaining unchanged. 
When DSP receives HALL signal, it needs to collect and process the output signal from each magnetic sensor and infrared obstacle avoidance sensor, so that the purpose of intelligent navigation can be achieved. Finally, IKCM30F60GA IPM intelligent power supply driver module is applied.

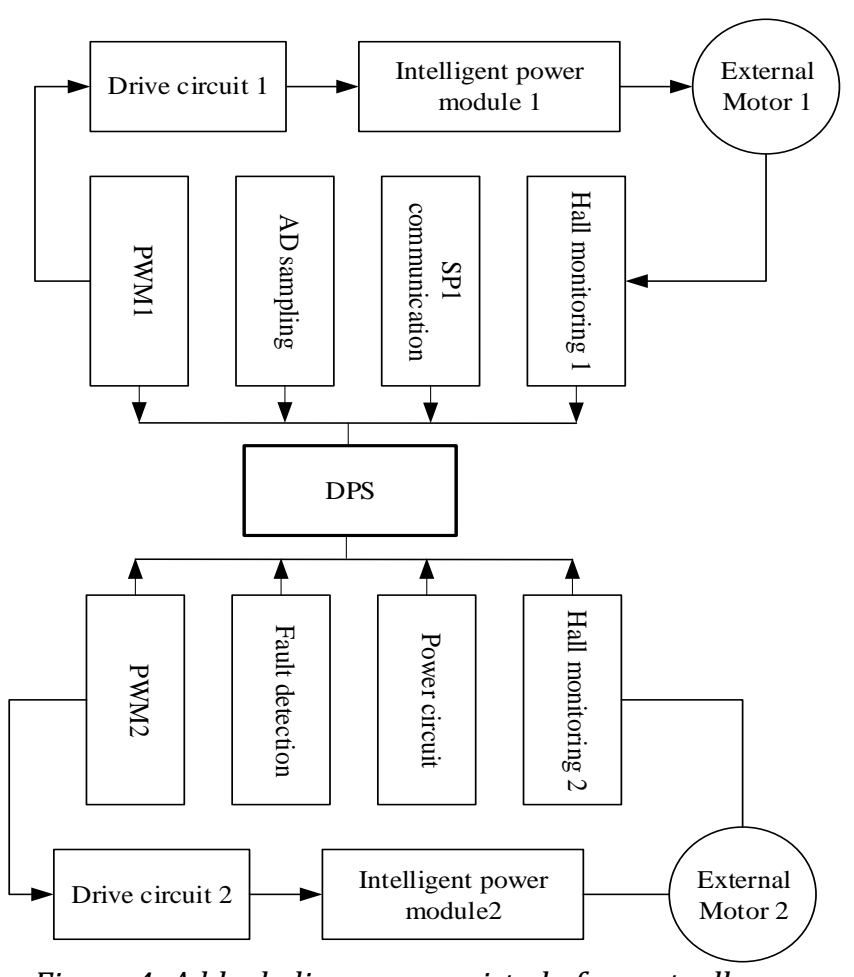

Figure 4: A block diagram consisted of a controller.

\subsection{The main control module algorithm of electronic differential speed system in intelligent logistics vehicle}

According to Ackerman's steering geometry principle, the main mathematical model of electronic differential system, the electronic differential model in this research is designed, as shown in Figure 5.

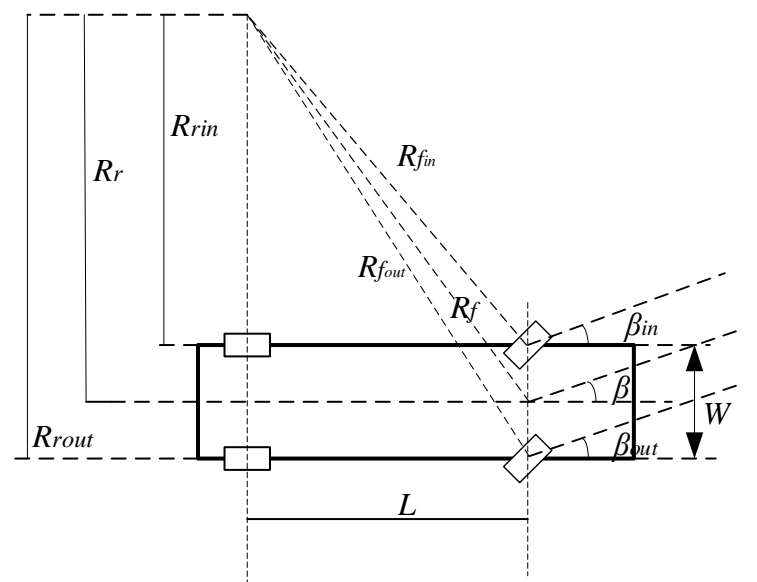

Figure 5: Ackerman's steering geometry principle based on electron differential velocity.

Assuming that the distance between the center of mass of the front and rear wheels of the logistics vehicle is $\mathrm{L}$, and the distance between the center of mass of the left and right wheels is the steering radius of the rear wheels, the steering radius of the inner wheels and the steering radius of the outer wheels $W$. In the figure, $R_{r}, R_{\text {rin }}$ and $R_{\text {rout }}$ are front wheel steering radius, inner wheel steering radius and outer wheel steering radius, respectively. $\beta_{\text {in, }}$ $\beta_{\text {out }}$ and $\beta$ represent the inner front wheel angle steering, the inner and outer wheel angle steering, and the steering Angle of the logistics vehicle. $C_{f i n}$ and $C_{\text {fout }}$ respectively represent the steering perimeter of the inner and outer wheels of the front wheel [14].

Then equations below are obtained.

$$
\begin{aligned}
& R=L / \tan \beta \\
& R_{\text {fout }}=R_{f}+W / 2 \\
& R_{\text {fout }}=R_{f}+W / 2 \\
& C_{\text {fout }}=2 \pi R_{\text {out }} \\
& C_{\text {fin }}=2 \pi R_{\text {fin }}
\end{aligned}
$$

Deduced from Eq. 1 to Eq. 5, if $K=\frac{\pi W}{\Delta t}$, then Eq. 6 and Eq. 7 are obtained.

$$
v_{f i n}=\frac{C_{f i n}}{\Delta t}=2 \pi \frac{R}{\Delta t}-\frac{\pi W}{\Delta t}=V_{f}+K
$$




$$
v_{\text {fout }}=\frac{C_{\text {fout }}}{\Delta t}=2 \pi \frac{R}{\Delta t}-\frac{\pi W}{\Delta t}=V_{f}+K
$$

In equations, $\Delta t$ represents the time required for the logistics vehicle to make one turn.

The required time in the logistics car in a round is $\Delta t$. However, since the driving motor used in this research is the hub motor, the speed of Wie motor multiplied by the coefficient is the actual speed. The time required for a turn can be obtained indirectly through the motor speed.

Finally, from Ackerman's geometric principle, the internal and external velocity equation is obtained.

$$
\begin{aligned}
& v_{\text {fout }}=v\left(1+\tan \beta_{\text {out }} \frac{W}{2 L}\right) \\
& v_{\text {fin }}=v\left(1-\tan \beta_{\text {in }} \frac{W}{2 L}\right)
\end{aligned}
$$

In equations, the equations of two steering angles of the inner and outer wheels are obtained.

$$
\begin{aligned}
& \beta_{\text {out }}=\arctan \left(\frac{L \tan \beta}{L+\frac{W}{2} \tan \beta}\right) \\
& \beta_{\text {in }}=\arctan \left(\frac{L \tan \beta}{L+\frac{W}{2} \tan \beta}\right)
\end{aligned}
$$

After calculating Eq. 8 and Eq. 9, the steering speed of the two steering front wheels is obtained.

After the signal is transmitted to the lower brushless DC motor controller, the wheel speed can be controlled in real time.

In order to reduce the vibration of the automatic guide vehicle, increase the stability of the automatic guide vehicle, and reduce the noise generated during the operation, a fuzzy PI regulator is introduced in the motor speed control.

\section{Results}

\subsection{No-load test results of outer rotor brushless DC motor}

Figure. 6 and Figure. 7 respectively show the noload electromagnetic torque waveform and the reverse electromotive force waveform of the external hub motor.

According to the waveform, the no-load back potential adopted in this research is $10 \mathrm{~V}$.

The three-phase current has a high symmetry, and it can be concluded from the electromagnetic torque waveform that the evaluation torque is $7 \mathrm{~N} \cdot \mathrm{m}$.

Therefore, it conforms to the design of the driving requirements mentioned above in the case of no load.

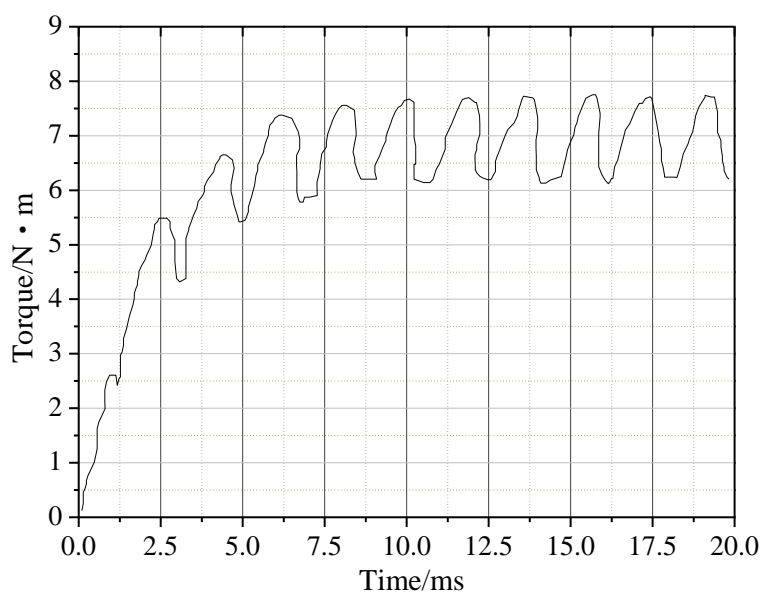

Figure 6: No-load electromagnetic torque waveform of external hub motor.

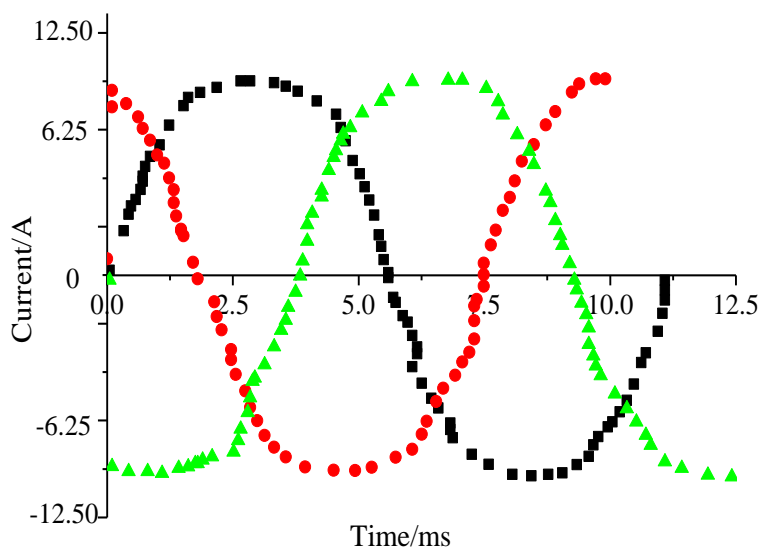

Figure 7: The no-load back electromotive force waveform of the external hub motor. 


\subsection{Experimental results of external rotor brushless DC motor load}

When carrying out experiments under load conditions, the full load mass of the AGV vehicle system designed in this research is $300 \mathrm{~kg}$.

Taking the strength of the body into consideration, the mass of the whole vehicle is about $150 \mathrm{~kg}$. Figure 8 is the variation diagram of motor load current in the experiment.

The three-phase current of the external motor is also symmetric, and the peak of the current is well suppressed during the phase conversion.

The current amplitude is about 5A, which is about half of the rated current of the motor.

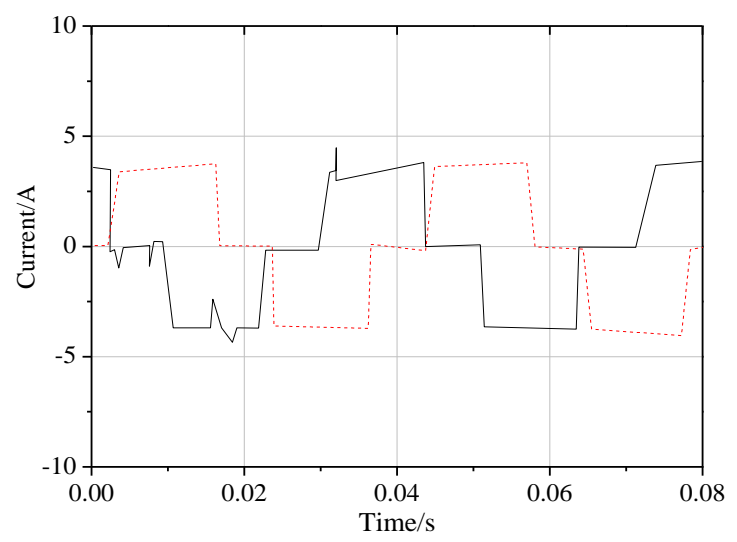

Figure 8: The load current of motor.

In this research, the electronic differential control of conventional PI in the speed link PI regulator is compared with the electronic differential control of fuzzy PI in the load experiment, as shown in Figure 9.

The response time of fuzzy PI electronic differential control system is shorter than that of conventional PI electronic differential control system, and fuzzy PI electronic differential control system has better anti-interference and stability than conventional PI electronic differential control system.

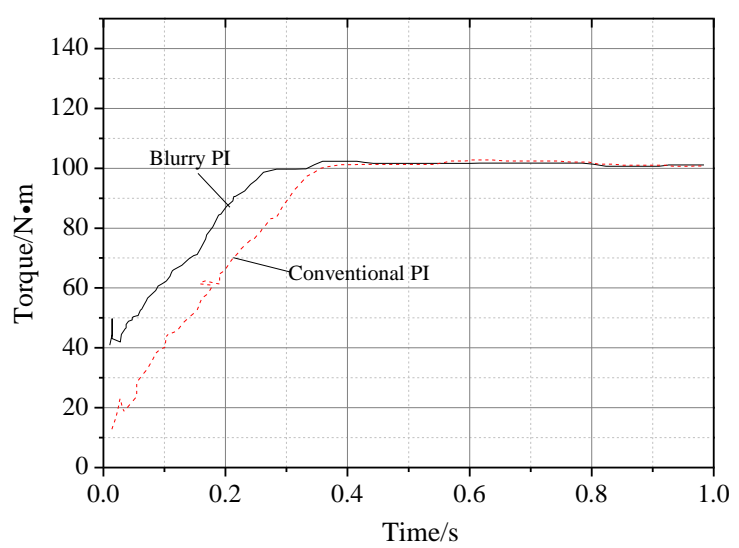

Figure 9: The corresponding comparison diagram between the conventional PI control and the fuzzy PI control motor speed.

\subsection{Electron differential steering test results}

In the differential steering experiment, CCF-GTN 30 tape is used.

Considering the site of the experiment in this research and the demand for the tape width, the tape with a thickness of $1.2 \mathrm{~mm}$ and a width of $30 \mathrm{~mm}$ is selected.

In order to verify the consistency of the left and right wheels of the intelligent logistics vehicle designed in this research when traveling in a straight line, a mass of $1 \mathrm{~m} / \mathrm{s}$ is given in the experiment.

The route of the collector's left and right wheels is compared, as shown in Figure 10. After the speed of the wheels on both sides of the left and right wheels increases from 0 to $1 \mathrm{~m} / \mathrm{s}$, the speed of the wheels keeps moving rapidly.

And the left and right wheels of the wheel basically keep the same form of distance, the routes are basically the same, However, when the driving distance of Figure 10 before and after $8 \mathrm{~s}$ is magnified for observation, it can be concluded that the actual driving speed of the AGV vehicle is less than $1 \mathrm{~m} / \mathrm{s}$ specified in the experiment.

The main reason for this is that logistics vehicles don't always keep a straight line in the process of driving.

This is mainly due to the fact that the straightness of the tape may be poor when the tape is laid in the straight path, or in the bumpy form of the route, the controller's control of the vehicle is influenced by the vehicle's erratic driving and stopping speed.

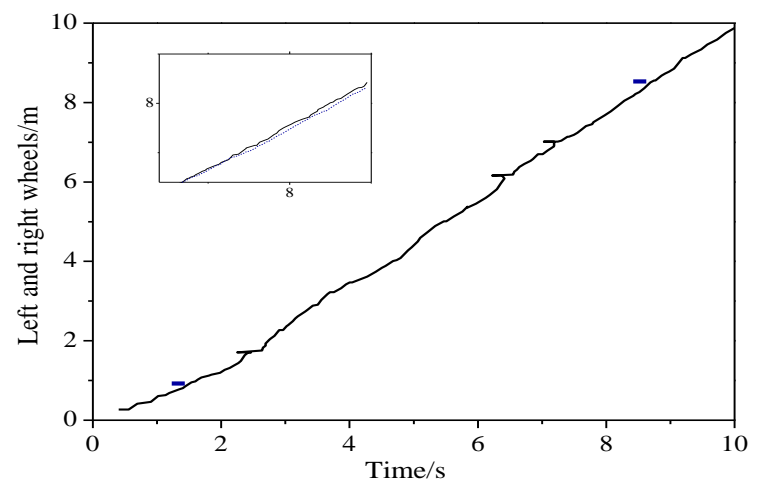

Figure 10: The number of miles that the AGV travels on both left and right wheels under the specified command.

In order to verify the accuracy of the electronic differential steering control strategy, instructions are given during the driving of the left and right-side wheels.

The command is executed within $0 \sim 3 \mathrm{~s}$, then turning left 30 degrees within $3 \sim 5.5$ seconds is carried out, and straight driving is kept for $5.5 \sim 8 \mathrm{~s}$. The process of driving routes is collected, according to the which, the difference is calculated.

Figure 11 shows the driving path of left and right-side wheels under differential speed command. 


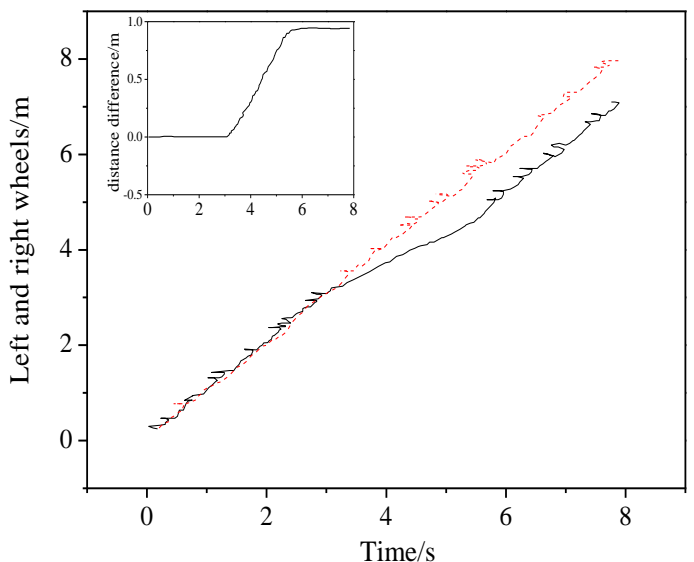

Figure 11: The difference diagram of the driving path of left and right-side wheels under differential speed instruction.

According to the difference diagram in the upper left corner of Figure 11, it can be concluded that within $0 \sim 3 \mathrm{~s}$, the driving distance difference between the left and right-side wheels under the straight-line command is 0 , which indicates that the left and rightside wheels have good consistency and correspondence during driving. In $3 \sim 5.5$, under the corner command, the wheel speed changes to $0.61 \mathrm{~m} / \mathrm{s}$, while the speed of the right wheel is $1 \mathrm{~m} / \mathrm{s}$. According to the difference, the actual angle is 28 degrees. According to the experiment, there is a big deviation between the experimental result and the target instruction in the experiment, which is mainly caused by two reasons. One reason is because there's a transition from going straight to turning when giving the command. Another reason is that the uneven road surface and wheel slippage don't respond to commands in a timely manner.

\section{Discussion}

In order to study the mechanical structure and automatic control of an intelligent logistics vehicle, an algorithm based on the main control module of electronic differential system is proposed to optimize the controller by taking the automatic guide vehicle as an example. And the fuzzy IP controller is designed. Firstly, in terms of hardware design, the hub motor, mechanical structure, and controller of intelligent logistics vehicle are designed. In the process of hub motor design, according to the driving requirements of the logistics vehicle designed in this research, the external permanent magnet brushless DC hub motor is adopted. In the design of mechanical structure, four wheels are selected, and the controller based on electronic differential velocity is selected. Then the software for the logistics vehicle is designed.

Firstly, the main control module algorithm of the electronic differential system is introduced, and then the external rotating electrode is controlled by fuzzy IP.
The idea of this research is basically consistent with that of literature [15]. In order to improve the performance of the intelligent logistics vehicle, it is necessary to design the hardware and software of the logistics vehicle respectively. Hardware design usually includes hub motor, mechanical structure, controller design, while software design basically includes vehicle control, external motor control and other parts. The performance of the logistics vehicle is improved through hardware and software optimization. According to the requirements of logistics vehicles, the hardware and software of logistics vehicles are designed respectively. The advantages of the design in this research are verified by experiments. The logistics vehicle designed in this research has better stability and accuracy, which are obtained from the no-load, load, and electronic differential speed experiments of the external rotor brushless DC motor. Similarly, a kind of intelligent mechanical structure automatic tracking guide vehicle is designed in literature [16]. Under the requirements of personalized design and target, the system can automatically realize the functions of tracking, carrying, classification and storage of goods.

\section{Conclusions}

In order to improve the performance of intelligent logistics vehicles in all aspects and further expand the application field of intelligent logistics vehicles, through the design of hardware and software of the intelligent logistics vehicle, the hub motor, mechanical structure and controller are designed respectively in terms of hardware.

Through comparative analysis, the external permanent magnet brushless DC hub motor is adopted, the four-wheel form and the controller based on electronic differential speed are selected. In terms of software, the controller is optimized based on the master control algorithm of electronic differential system.

Then the external rotating electrode is controlled by fuzzy IP. Finally, through the experiment of no-load, load and electronic differential steering of the external transmission sub-brushless motor, the feasibility of the logistics vehicle based on the electronic differential model is obtained

The research has made an important contribution to the logistics development of ecommerce industry in China, but it still has limitations. It doesn't analyze and experiment the guiding design of logistics vehicle.

It is hoped that the guiding design can be studied in the follow-up research, and the gap in this study can be supplemented to make the research more comprehensive. 


\section{References}

[1] Li G., Lin R., Li M, et al. (2019) A Master-Slave Separate Parallel Intelligent Mobile Robot Used for Autonomous Pallet Transportation [J]. Applied Sciences, 9(3), 368.

[2] Yang Y., Zhong M., Yao H, et al. (2018) Internet of things for smart ports: Technologies and challenges [J]. IEEE Instrumentation \& Measurement Magazine, 21(1), 34-43.

[3] Tran A. S., Ngo H. Q. T. (2018) Research and manufacture of automated guided vehicle for the service of storehouse [J]. Science and Technology Development Journal-Engineering and Technology, 1(1), 5-12.

[4] Kłosowski G., Gola A., Amila T. (2018) Computational intelligence in control of AGV multimodal systems [J]. Ifac-papersonline, 51(11), 1421-1427.

[5] Kaliappan S., Lokesh J., Mahaneesh P, et al. (2018) Mechanical Design and Analysis of AGV for Cost Reduction of Material Handling in Automobile Industries[J]. International Research Journal of Automotive Technology, 1(1), 1-7.

[6] Gao Z., Du Y., Zhang Y, et al. (2015) Design and Development on Automatic Guided Logistics Platform with Robotic Arm [J]. Machinery Design \& Manufacture, (8), 41.

[7] Peng T., Qian J., Zi B, et al. (2016) Mechanical design and control system of an omni-directional mobile robot for material conveying[J]. Procedia CIRP, 56, 412-415.

[8] Zhang J., Zhang H., Zhao Y, et al. (2018) Design for Micro Intelligent Automatic Tracking AGV Based on Arduino Microcontroller [J]. Machine Tool \& Hydraulics, (3), 12.
[9] Wu X., Wu J., Jin P, et al. (2018) Genetic Algorithm-Based Structure Optimization and Load-Carrying Analysis for the Body Frame of Heavy-Duty AGVs [J]. International Journal of Robotic Engineering, 3(2), 1-10.

[10] Li G., Lin R., Li M, et al. (2019) A Master-Slave Separate Parallel Intelligent Mobile Robot Used for Autonomous Pallet Transportation [J]. Applied Sciences, 9(3), 368.

[11] Tran A. S., Ngo H. Q. T. (2018) Research and manufacture of automated guided vehicle for the service of storehouse [J]. Science and Technology Development Journal-Engineering and Technology, 1(1), 5-12.

[12] Jacobs L., De Preter A., Anthonis J, et al. (2018) Trajectory tracking of AGVs by linear parameter-varying control: a case study[J]. IFAC-Papers On Line, 51(26), 43-48.

[13] Yao J., Chen J., Wang Q. (2018) Design and realization of a three-dimensional garage control and management system based on agv carrier [J]. Mechanical Engineering \& Automation, (1), 75.

[14] Mehami J., Nawi M., Zhong R. Y. (2018) Smart automated guided vehicles for manufacturing in the context of Industry 4.0[J]. Procedia Manufacturing, 26, 1077-1086.

[15] Wang H., Guan H., Wang P, et Al. (2019) Design of camera tracking car based on ROS system [J]. Information Technology and Informatization, (2), 35.

[16] Hang P., Chen X., Zhang B, et al. (2018) Longitudinal velocity tracking control of a 4WID electric vehicle [J]. IFAC-Papers On Line, 51(31), 790-795. 